\title{
GROTHENDIECK'S THEOREM FOR ABSOLUTELY SUMMING MULTILINEAR OPERATORS IS OPTIMAL
}

\author{
D. PELLEGRINO AND J. B. SEOANE-SEPÚLVEDA
}

\begin{abstract}
Grothendieck's theorem asserts that every continuous linear operator from $\ell_{1}$ to $\ell_{2}$ is absolutely $(1 ; 1)$-summing. In this note we prove that the optimal constant $g_{m}$ so that every continuous $m$-linear operator from $\ell_{1} \times \cdots \times \ell_{1}$ to $\ell_{2}$ is absolutely $\left(g_{m} ; 1\right)$-summing is $\frac{2}{m+1}$. We also show that if $g_{m}<\frac{2}{m+1}$ there is $\mathfrak{c}$ dimensional linear space composed by continuous non absolutely $\left(g_{m} ; 1\right)$-summing $m$-linear operators from $\ell_{1} \times \cdots \times \ell_{1}$ to $\ell_{2}$. In particular, our result solves (in the positive) a conjecture posed by A.T. Bernardino in 2011.
\end{abstract}

\section{INTRODUCTION}

A celebrated result of Grothendieck asserts that every continuous linear operator from $\ell_{1}$ to $\ell_{2}$ is absolutely $(1 ; 1)$-summing. It was recently proved 3 that this result can be lifted to multilinear operators in the following fashion:

$$
\begin{aligned}
& \text { Every continuous m-linear operator from } \ell_{1} \times \cdots \times \ell_{1} \text { to } \ell_{2} \text { is absolutely }\left(\frac{2}{m+1} ; 1\right) \text { - } \\
& \text { summing. }
\end{aligned}
$$

In the same paper the author conjectured that the value $\frac{2}{m+1}$ is optimal. A particular case of our main result gives a positive solution to this conjecture:

Theorem 1.1. The estimate $\frac{2}{m+1}$ is optimal. Moreover, if $g_{m}<\frac{2}{m+1}$ then there exists a $\mathfrak{c}$ dimensional linear space formed (except by the null vector) by continuous non absolutely $\left(g_{m} ; 1\right)$ summing m-linear operators. This result is optimal in terms of dimension.

Above, $\mathfrak{c}$ denotes the cardinality of the continuum. In other words, our main result shows that if $g_{m}<\frac{2}{m+1}$, the set of continuous non absolutely $\left(g_{m} ; 1\right)$-summing multilinear operators is $\mathfrak{c}$-lineable and moreover, maximal lineable. For the theory of lineability we refer to [1, 2] and the references therein.

Our proof of the optimality of $\frac{2}{m+1}$ is inspired on ideas that date back to the classical work of Lindenstrauss and Pełczyński [9] and, later, explored in a series of papers (see, e.g., 4, 5, 6, 10]).

Throughout this note, $X$ and $Y$ shall stand for Banach spaces over $\mathbb{K}=\mathbb{R}$ or $\mathbb{C}$. The closed unit ball of $X$ is denoted by $B_{X}$ and the topological dual of $X$ by $X^{*}$. Also, recall that a continuous linear operator $u: X \rightarrow Y$ is absolutely $(q ; 1)$-summing (see [7]) if there exists $C \geq 0$ such that

$$
\left(\sum_{j=1}^{n}\left\|u\left(x_{j}\right)\right\|^{q}\right)^{\frac{1}{q}} \leq C \sup _{\varphi \in B_{X^{*}}} \sum_{j=1}^{n}\left|\varphi\left(x_{j}\right)\right|
$$

for every $n \in \mathbb{N}$ and $x_{1}, \ldots, x_{n} \in X$. The nonlinear theory of absolutely summing operators was designed by Pietsch in 1983 ([1]) and since then has been intensively studied. One of the possible polynomial generalizations of absolutely summing operators is the concept of absolutely summing polynomial. The space of continuous $m$-homogeneous polynomials from $X$ to $Y$ will be henceforth denoted by $\mathcal{P}\left({ }^{m} X ; Y\right)$. Given a positive integer $m$ and $q \geq \frac{1}{m}$, a continuous $m$ homogeneous polynomial $P: X \rightarrow Y$ is absolutely $(q ; 1)$-summing if there exists a constant

Key words and phrases. lineability, spaceability, absolutely summing operators. 
$C \geq 0$ such that

$$
\left(\sum_{j=1}^{n}\left\|P\left(x_{j}\right)\right\|^{q}\right)^{\frac{1}{q}} \leq C\left(\sup _{\varphi \in B_{X^{*}}} \sum_{j=1}^{n}\left|\varphi\left(x_{j}\right)\right|\right)^{m}
$$

for every $n \in \mathbb{N}$ and $x_{1}, \ldots, x_{n} \in X$. If $q<1$, the infimum of the constants $C$ satisfying the above inequality is a Banach quasinorm for the space of absolutely $(q, 1)$-summing polynomials from $X$ to $Y$, and it is denoted by $\pi_{q, 1}$. For multilinear mappings the definition is similar:

A continuous $m$-linear operator $T: X \times \cdots \times X \rightarrow Y$ is absolutely $(q ; 1)$-summing (with $q \geq \frac{1}{m}$ ) if there is a constant $C \geq 0$ such that

$$
\left(\sum_{j=1}^{n}\left\|T\left(x_{j}^{(1)}, \ldots, x_{j}^{(m)}\right)\right\|^{q}\right)^{\frac{1}{q}} \leq C \prod_{k=1}^{m}\left(\sup _{\varphi \in B_{X^{*}}} \sum_{j=1}^{n}\left|\varphi\left(x_{j}^{(k)}\right)\right|\right)
$$

for every $n \in \mathbb{N}$ and $x_{1}^{(k)}, \ldots, x_{n}^{(k)} \in X$, and $k=1, \ldots, m$. If $q<1$, the infimum of the constants $C$ satisfying the above inequality is a Banach quasinorm for the space of absolutely $(q ; 1)$-summing $m$-linear operators from $X \times \cdots \times X$ to $Y$.

\section{The Proof of Theorem 1.1}

Let $1 \leq g_{m}<\frac{2}{m+1}$. The first part of our argument is mentioned en passant, without proof, in [6], but since we have a more self-contained approach, we present the details for the sake of completeness. Let $n \in \mathbb{N}$ and $x_{1}, \ldots, x_{n} \in \ell_{1}$ be non null vectors. Consider $x_{1}^{*}, \ldots, x_{n}^{*} \in B_{\ell_{\infty}}$ so that $x_{j}^{*}\left(x_{j}\right)=\left\|x_{j}\right\|$ for every $j=1, \ldots, n$. Let $a_{1}, \ldots, a_{n}$ be scalars such that $\sum_{j=1}^{n} \mid a_{j} \frac{2}{g_{m}}=1$ and define

$$
P_{n}: \ell_{1} \longrightarrow \ell_{2}, P_{n}(x)=\sum_{j=1}^{n}\left|a_{j}\right|^{\frac{1}{g_{m}}} x_{j}^{*}(x)^{m} e_{j},
$$

where $e_{j}$ is the $j$-th canonical vector of $\ell_{2}$. For every $x \in \ell_{1}$,

$$
\left\|P_{n}(x)\right\|=\left(\left.\left.\sum_{j=1}^{n}|| a_{j}\right|^{\frac{1}{g_{m}}} x_{j}^{*}(x)^{m}\right|^{2}\right)^{\frac{1}{2}} \leq\left(\sum_{j=1}^{n}\left|a_{j}\right|^{\frac{2}{g_{m}}}\right)^{\frac{1}{2}}\|x\|^{m}=\|x\|^{m} .
$$

Since $P_{n}$ is a polynomial of finite type, then it is plain that $P_{n}$ is absolutely $\left(g_{m} ; 1\right)$-summing. Note that for $k=1, \ldots, n$, we have

$$
\left\|P_{n}\left(x_{k}\right)\right\|=\left\|\sum_{j=1}^{n}\left|a_{j}\right|^{\frac{1}{g_{m}}} x_{j}^{*}\left(x_{k}\right)^{m} e_{j}\right\| \geq\left|a_{k}\right|^{\frac{1}{g_{m}}} x_{k}^{*}\left(x_{k}\right)^{m}=\left|a_{k}\right|^{\frac{1}{g_{m}}}\left\|x_{k}\right\|^{m} .
$$

To simplify the notation we write $\left\|\left(x_{j}\right)_{j=1}^{n}\right\|_{w, 1}:=\sup _{\varphi \in B_{X^{*}}} \sum_{j=1}^{n}\left|\varphi\left(x_{j}^{(k)}\right)\right|$. Thus, we have

$$
\begin{aligned}
\left(\sum_{j=1}^{n}\left\|x_{j}\right\|^{m g_{m}}\left|a_{j}\right|\right)^{\frac{1}{g_{m}}} & =\left(\sum_{j=1}^{n}\left(\left\|x_{j}\right\|^{m} \mid a_{j} \frac{1}{g_{m}}\right)^{g_{m}}\right)^{\frac{1}{g_{m}}} \\
& \leq\left(\sum_{j=1}^{n}\left\|P_{n}\left(x_{j}\right)\right\|^{g_{m}}\right)^{\frac{1}{g_{m}}} \\
& \leq \pi_{g_{m}, 1}\left(P_{n}\right)\left\|\left(x_{j}\right)_{j=1}^{n}\right\|_{w, 1}^{m} .
\end{aligned}
$$


Since this last inequality holds whenever $\sum_{j=1}^{n}\left|a_{j}\right|^{\frac{2}{g_{m}}}=1$, denoting $\left(\frac{2}{g_{m}}\right)^{*}$ to the conjugate of $\frac{2}{g_{m}}$ we obtain

$$
\begin{aligned}
\left(\sum_{j=1}^{n}\left\|x_{j}\right\|^{m g_{m}\left(\frac{2}{g_{m}}\right)^{*}}\right)^{\frac{1}{\left(\frac{2}{g_{m}}\right)^{*}}} & \leq \sup \left\{\sum_{j=1}^{n}\left|a_{j}\right|\left\|x_{j}\right\|^{m g_{m}} ; \sum_{j=1}^{n}\left|a_{j}\right|^{\frac{2}{g_{m}}}=1\right\} \\
& \leq\left(\pi_{g_{m}, 1}\left(P_{n}\right)\left\|\left(x_{j}\right)_{j=1}^{n}\right\|_{w, 1}^{m}\right)^{g_{m}}
\end{aligned}
$$

and, then,

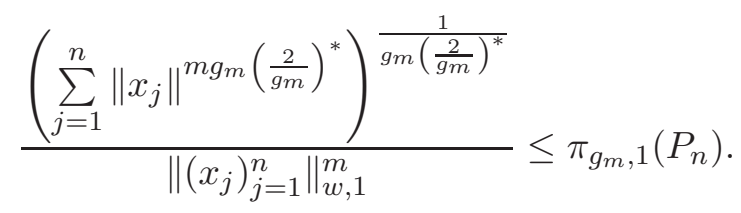

Since $1 \leq g_{m}<\frac{2}{m+1}$ we have $m g_{m}\left(\frac{2}{g_{m}}\right)^{*}<2$ and from a weak version of the Dvoretzky-Rogers Theorem we know that $i d_{\ell_{1}}$ is not $\left(m g_{m}\left(\frac{2}{g_{m}}\right)^{*} ; 1\right)$-summing. Combining this fact with (1D) we conclude that we can find $x_{j}$ in $\ell_{1}$ for all positive integer $j$ so that

$$
\lim _{n \rightarrow \infty} \pi_{g_{m}, 1}\left(P_{n}\right)=\infty \text { and }\left\|P_{m}\right\|=1 .
$$

We thus conclude that the space of all absolutely $\left(g_{m} ; 1\right)$-summing $m$-homogeneous polynomials from $\ell_{1}$ to $\ell_{2}$ is not closed in $\mathcal{P}\left({ }^{m} \ell_{1} ; \ell_{2}\right)$. In fact, otherwise, since the quasinorm $\pi_{g_{m}, 1}$ is complete, the Open Mapping Theorem to $F$-spaces would contradict (2).

Now, let $P: \ell_{1} \rightarrow \ell_{2}$ be a continuous non $\left(g_{m} ; 1\right)$-summing $m$-homogeneous polynomial. Split $\mathbb{N}$ into a countable union of pairwise disjoint countable sets $\mathbb{N}_{1}, \mathbb{N}_{2}, \ldots$. For all $j$, let

$$
\mathbb{N}_{j}=\left\{a_{1}^{(j)}<a_{2}^{(j)}<\cdots\right\}
$$

and define $P^{(j)}: \ell_{1} \rightarrow \ell_{2}$ by $\left(P^{(j)}(x)\right)_{a_{k}^{(j)}}=(P(x))_{k}$ and $\left(P^{(j)}(x)\right)_{k}=0$ if $k \notin \mathbb{N}_{j}$. It is simple to prove that $P^{(j)}$ is also a continuous non $\left(g_{m} ; 1\right)$-summing $m$-homogeneous polynomial and the set $\left\{P^{(1)}, P^{(2)}, \ldots\right\}$ is linearly independent. Finally, we note that the linear operator $\Phi: \ell_{1} \rightarrow \mathcal{P}\left({ }^{n} \ell_{1} ; \ell_{2}\right)$ given by $\left(\beta_{j}\right)_{j=1}^{\infty} \mapsto \sum_{j=1}^{\infty} \beta_{j} P^{(j)}$ is injective and it is simple to verify that $\Phi\left(\ell_{1}\right)$ is composed (except by the null vector) exclusively by non absolutely $\left(g_{m} ; 1\right)$-summing $m$-homogeneous polynomials. We thus conclude that the set of continuous non $\left(g_{m} ; 1\right)$-summing $m$-homogeneous polynomials is $\Phi\left(\ell_{1}\right)$-lineable. Since $\operatorname{dim} \Phi\left(\ell_{1}\right)=\operatorname{dim} \ell_{1}=\mathfrak{c}$ we conclude that this set is $\mathfrak{c}$-lineable.

Since $\mathcal{P}\left({ }^{n} \ell_{1} ; \ell_{2}\right)$ is isomorphic to the space of symmetric $m$-linear operators from $\ell_{1} \times \cdots \times \ell_{1}$ to $\ell_{2}$ and since $P$ is absolutely $\left(g_{m} ; 1\right)$-summing if and only if its associated symmetric $m$-linear operator is absolutely $\left(g_{m} ; 1\right)$-summing, our result is translated to the multilinear setting.

The above result is optimal in terms of dimension. In fact, it is well known that $\ell_{1}$ is isometric to the completion of its projective tensor product, i.e., $\ell_{1}=\ell_{1} \widehat{\otimes}_{\pi} \cdots \widehat{\otimes}_{\pi} \ell_{1}$. Thus

$$
\operatorname{dim} \mathcal{L}\left({ }^{m} \ell_{1} ; \ell_{2}\right)=\operatorname{dim} \mathcal{L}\left(\ell_{1} \widehat{\otimes}_{\pi} \cdots \widehat{\otimes}_{\pi} \ell_{1} ; \ell_{2}\right)=\operatorname{dim} \mathcal{L}\left(\ell_{1} ; \ell_{2}\right)=\mathfrak{c} .
$$

We remark that if $\pi_{g_{m}, 1}$ was locally convex, since we have proved that the space of all absolutely $\left(g_{m} ; 1\right)$-summing $m$-homogeneous polynomials from $\ell_{1}$ to $\ell_{2}$ is not closed in the space of all continuous $m$-homogeneous polynomials from $\ell_{1}$ to $\ell_{2}$, then from a result due to Drewnowski (see [8, Theorem 5.6 and its reformulation ]) we would conclude that the set of all continuous $m$ homogeneous polynomials from $\ell_{1}$ to $\ell_{2}$ that fail to be absolutely $\left(g_{m} ; 1\right)$-summing is spaceable, i.e., contains (except for the null vector) a closed infinite-dimensional subspace. 


\section{REFERENCES}

[1] R.M. Aron, V. I. Gurariy, and J.B. Seoane-Sepúlveda, Lineability and spaceability of sets of functions on $\mathbb{R}$. Proc. Amer. Math. Soc. 133, 795-803 (2005)

[2] L. Bernal-González, D. Pellegrino and J.B. Seoane-Sepúlveda, Linear subsets of nonlinear sets in topological vector spaces. Bull. Amer. Math. Soc. (N.S.) 51 (2014), no. 1, 71-130.

[3] A.T. Bernardino, On cotype and a Grothendieck-type theorem for absolutely summing multilinear operators, Quaest. Math. 34 (2011), 513-519.

[4] G. Botelho and D. Pellegrino, Scalar-valued dominated polynomials on Banach spaces, Proc. Amer. Math. Soc. 134 (2006), 1743-1751.

[5] G. Botelho and D. Pellegrino, Absolutely summing polynomials on Banach spaces with unconditional basis, J. Math. Anal. Appl. 321 (2006), 50-58.

[6] G. Botelho, D. Pellegrino, and P. Rueda, Cotype and absolutely summing linear operators. Math. Z. 267 (2011), no. 1-2, 1-7.

[7] J. Diestel, H. Jarchow, and A. Tonge, Absolutely summing operators, Cambridge University Press, 1995.

[8] L. Drewnowski, Quasicomplements in F-spaces, Studia Math. 77 (1984) 373-391.

[9] J. Lindenstrauss and A. Pełczyński, Absolutely summing operators in $\mathcal{L}_{p}$ spaces and their applications, Studia Math. 29 (1968), 275-326.

[10] D. Pellegrino, Cotype and absolutely summing homogeneous polynomials in $\mathcal{L}_{p}$ spaces, Studia Math. 157 (2003), 121-131.

[11] A. Pietsch, Ideals of Multilinear Functionals. Proc. II Intern. Conf. on Operator Algebras, Ideals and Appl. in Theoretical Physics. Leipzig (1983) Teubner-Texte.

Departamento de Matemática,

Universidade Federal da PARAíba,

58.051-900 - Jỗo PessoA, Brazil.

E-mail address: dmpellegrino@gmail.com and pellegrino@pq.cnpq.br

Departamento de Análisis Matemático,

Facultad de Ciencias Matemáticas,

Plaza de Ciencias 3,

Universidad COMPlutense de Madrid,

MADRID, 28040, SPAIN.

E-mail address: jseoane@mat.ucm.es 